\title{
Celestial dynamics and astrometry in an expanding universe
}

\author{
S. Kopeikin \\ Department of Physics \& Astronomy, University of Missouri, 322 Physics Building, \\ Columbia, MO 65211, USA
}

\begin{abstract}
The mathematical concept of the Newtonian limit of Einstein's equations in an expanding universe is formulated. The equations of motion of planets and light are compared.
\end{abstract}

Keywords. gravitation, relativity, ephemerides, cosmology: theory

The current paradigm of the IAU 2000 resolutions assumes that the background spacetime is flat with the Minkowski metric tensor $\eta_{\alpha \beta}$. A more adequate model is the Friedmann spacetime with the metric $\bar{g}_{\alpha \beta}=a^{2}(\eta) \eta_{\alpha \beta}$, where $a(\eta)$ is the scale factor describing the Hubble expansion. The coordinate chart is $x^{\alpha}=\left(c \eta, x^{i}\right)$, where the conformal (non-physical) time $\eta$ relates to the cosmic (physical) time $t$ measured by the Hubble observers, by the equation $a(\eta) d \eta=d t$. We assume that $\eta=0$ corresponds to the present epoch. The solar system is a localized astronomical system. It makes sense to introduce the local coordinates, $r^{\alpha}=\left(c \lambda, r^{i}\right)$, related to the global coordinates, $x^{\alpha}$, by a special conformal transformation, $r^{\alpha}=\left(x^{\alpha}-b^{\alpha} x^{2}\right) / \sigma(x)$, where $b^{\alpha}$ is a constant vector, $\sigma(x)=1-2 b_{\mu} x^{\mu}+x^{2}$, and $x^{2} \equiv \eta_{\mu \nu} x_{\mu} x^{\nu}, b^{2} \equiv \eta_{\mu \nu} b_{\mu} b^{\nu}$ (Fulton et al. 1962). The vector $b^{\alpha}$ is chosen so that $a^{2}(\eta) \eta_{\alpha \beta} d x^{\alpha} d x^{\beta}=\eta_{\alpha \beta} d r^{\alpha} d r^{\beta}$ is a valid equation up to the second order in the Hubble constant, $H=\dot{a} / a$. Transformation to the local metric is achieved with $b^{\alpha}=(H / 2 c) \bar{u}^{\alpha}$, where $\bar{u}^{\alpha}$ is four-velocity of the Hubble flow. Approximating $a(\eta)=1+H \eta$ yields, $\lambda=a(\eta)\left[\eta-(1 / 2) H\left(c^{2} \eta^{2}-\boldsymbol{x}^{2}\right)\right]$, and $\boldsymbol{r}=a(\eta) \boldsymbol{x}$. We have to relate the coordinate time $\lambda$ to the cosmic time $t$. In the linearized approximation $\eta=t-(1 / 2) H t^{2}$. For particles at rest, the conformal transformation of time yields $\lambda=t$. However, light moves along null geodesics, $c^{2} \eta^{2}-\boldsymbol{x}^{2}=0$. The conformal time transformation yields the relation of parameter $\lambda$ of the light geodesics with the cosmic time, $\lambda=t+(1 / 2) H t^{2}$. It means that in the Newtonian limit the metric for slowly-moving particles is $\eta_{\alpha \beta}$ but the optical metric for light is $\hat{g}_{\alpha \beta}=\eta_{\alpha \beta}+\left(1-a^{2}\right) \bar{u}_{\alpha} \bar{u}_{\beta}$, and depends on the scale factor, $a=a(t)$. This is interpreted as an index of refraction, $n=1 / a$, that makes the coordinate speed of light, $c_{l}=c / n=a c$, growing as time goes from past to future, and decreases otherwise. The equations of motion of particles and light are reduced to their Newtonian form for both metrics. However, equations for particles and for light differ by terms of the first order in the Hubble constant (Kopeikin 2012). This leads to the important conclusion that the correct light-propagation equation for ephemeris calculations is $\lambda_{2}-\lambda_{1}=(1 / c)\left|\boldsymbol{r}_{2}-\boldsymbol{r}_{1}\right|$. It eliminates the so-called Pioneer anomaly (Anderson et al. 2002) which may really have a cosmological origin.

\section{References}

Fulton, T., Rohrlich, F., \& Witten, L. 1962, Rev. Mod. Phys., 34, 442

Kopeikin, S. 2012, Phys.Rev., D86, 064004

Anderson, J D., Laing, P. A., Lau, E. L., Liu, A. S., Nieto, M. M., \& Turyshev, S. G. 2002, Phys.Rev, D65, 082004 